\title{
ON PARTLY ORDERED FIELDS
}

\author{
D. W. DUBOIS ${ }^{1}$
}

1. Introduction. By a partly ordered field we shall mean a commutative field $F$ containing a nonempty subset $P$ closed under addition and division. $P$ is called the positive cone and is always closed under multiplication. If for every $x \in F$ either $x \in P,-x \in P$ or $x=0$ holds, then $F$ is ordered (in the familiar sense) or simply ordered. The relations " $x>y$," or " $y<x$ " mean that $x-y$ belongs to $P$.

An algebraic characterization of ordered fields was given by Artin and Schreier [2] in 1926 and by Reinhold Baer [3] in 1927, namely $F$ has an ordering if and only if the equation $\sum a_{i}^{2}=-1$ cannot be satisfied for $a_{i} \in F$, i.e., $F$ is formally real. The proof starts with the fact that in a formally real field the set of sums of nonzero squares is a positive cone, as defined above. Artin [1] also showed that this set consists of all elements which are positive under every possible ordering. In the "ordering in the large" or "ordering modulo a null class $N$ " defined by Baer [3] the set of positive elements is closed under addition and multiplication, but not under division. A field ordered modulo the null class $N$ has a simple ordering and $N$ is the set of all elements infinitesimal relative to a subfield. In 1940, Stone [6] stated a theorem characterizing the ring of all continuous real-valued functions on a compact Hausdorff space as a partly ordered ring. This theorem, especially in the form given by Kadison [4], very much simplifies our theory of partly ordered fields.

The principal results of this paper establish the structure of a partly ordered field $F$ satisfying the property (AC): If $x+1 / n$ is positive for $n=1,2, \cdots$, then $x \geqq 0$. We show that the positive cone $P$ of $F$ is the intersection of a family of positive cones belonging to simple orderings, i.e., simple cones. The family of orderings is split into equivalence classes and the classes are taken as points of a compact Hausdorff space; $F$ is the field of quotients of a domain $B$, where $B$

Presented to the Society, November 23, 1951 under the title Partly ordered fields, on September 3, 1952 under the title Structure of Archimedean partly ordered fields and on April 25, 1953 under the title Primitivity, strict reality, and the condition of Clifford in partly ordered fields; received by the editors April 23, 1955.

1 This paper was begun as a doctoral dissertation at the University of Oklahoma under the direction of Casper Goffman and A. A. Grau. 
is represented as a ring of continuous real-valued functions on this space.

Examples of partly ordered fields: (1) Let $F$ be the field $K(x)$ of rational functions over the field $K$ of all rational numbers, with $P$ the set of all functions that assume no negative values for real $x . F$ is (AC). (2) Replace $K$ in example 1 by the field $R$ of all real numbers. This also gives an (AC) field. (3) Let $F=K(x, i)$ where $i^{2}=-1$, and let $K(x)$ be simply ordered so that $x$ is positive and infinitesimal. Now let $a(x)+i b(x)$ belong to $P$ if $a(x)$ is positive in $K(x)$ and if $b(x) / a(x)$ is infinitesimal in $K(x)$. The field is not (AC).

If $\beta=\left[P_{\beta}\right]$ is a family of positive cones in the field $F$, then $\cap P_{\beta}$ is a positive cone. The partial ordering defined by the intersection will be called the product of the partial orderings defined by the $P_{\beta}$. For example, the positive cone $P$ given above for $K(x)$ is the intersection of all possible simple cones, or the partial ordering is the product of all simple orderings.

Every field of characteristic zero has a partial order defined by taking the set of positive rationals for $P$, but for any field other than the field of rationals, such a partial order is trivial. If $F$ has a positive cone $P$, and $G$ is the intersection of all subfields containing $P$, then the structure of $G$ determines that of $F$ except for the purely fieldtheoretic problem of the extension from $G$ to $F$. Accordingly we will be restricted to primitive partial orderings, i.e., those in which no proper subfield contains the positive cone. The field $G$ above has a primitive partial order induced by the partial order of $F$. Even so, we prove that every field of characteristic zero, except for nonformally-real algebraic extensions of the rationals, has at least one primitive partial order.

If $-n<x<n$ holds for some positive integer $n$, then $x$ is said to be bounded and the letter $B$ designates the set of all such elements. If $-1 / n<x<1 / n$ holds for $n=1,2,3, \cdots$, then $x$ is called infinitesimal and the set of all such elements is designated by $J$. As in the case of an ordered field, $B$ is a ring, $J$ is an ideal in $B$ (but not necessarily a prime ideal), and $B / J$ satisfies the reality condition: $\sum x_{t}^{2}=0$ implies all $x_{i}$ are zero.

2. Structure of the ring $B / J$. In this section the ring $B / J$ is shown to have a representation as a ring of real-valued continuous functions on a compact Hausdorff space. First we list some trivial properties of a partly ordered field $F: F$ contains the field of rationals in natural order; $F$ is a partly ordered group under addition; from $x>y$ and $p>0$ follows $p x>p y ; p>q>0$ implies $1 / q>1 / p>0$; if $-a<x<a$ and $-b<y<b$ then $-a b<x y<a b$ (this follows from the identities 


$$
\begin{aligned}
& a b+x y=\frac{1}{2}(a-x)(b-y)+\frac{1}{2}(a+x)(b+y), \\
& \left.a b-x y=\frac{1}{2}(a+x)(b-y)+\frac{1}{2}(a-x)(b+y)\right) .
\end{aligned}
$$

This last property implies that $B$ is a ring and that $J$ is an ideal in $B$. The following theorem is less trivial.

THEOREM 2.1. A necessary and sufficient condition that the partial order of $F$ be primitive is that $F$ be the field of quotients of $B$.

Proof. Let $F$ be primitive and let $p$ be an arbitrary positive element. It is easy to see that $1 /(1+p)$ and $1-1 /(1+p)$ are positive elements of $B$ so that

$$
p=(1-1 /(1+p)) /(1 /(1+p))
$$

belongs to the field of quotients $B^{\prime}$ of $B$. Since $F$ is primitive, and the field of quotients of $B$ contains every positive element of $F$, it follows that $B^{\prime}=F$.

Conversely, let $F=B^{\prime}$ and suppose $P \subset G$ where $G$ is a subfield of $F$. If $x$ is any element of $B$ then there is a positive integer $n$ with $n-x$ positive. Hence $G$ contains $n-(n-x)=x$. Consequently $G$ contains $B^{\prime}=F$, so that $F$ is primitive.

It is natural to inquire whether a partly ordered field $F$ may be a lattice. Answer: If $F$ is a lattice it is simply ordered. For let $x$ be a nonzero, non-negative element of $F$, assuming that $F$ is a lattice. There exist positive elements $p$ and $q$ such that $q+x$ is positive, and $p$ is the least upper bound of $x$ and zero. Set $t=p(q+x) /(p+q)>0$. Since $t-x=q(p-x) /(p+q) \geqq 0$, and $p$ is the least upper bound of $x$ and zero, we have $t \geqq p$. On the other hand, $t \leqq p$, since

$$
p-t=p(p-x) /(p+q) \geqq 0 .
$$

Hence $t=p$; consequently $x=p>0$, whence $F$ is simply ordered.

The weaker condition that $F$ be a directed set, i.e., for arbitrary $a$ and $b$ there is an $x$ such that $x>a, x>b$, holds in many partly ordered fields that are not simply ordered. Equivalent conditions are: every element is exceeded by at least one positive element; and no proper subring contains the positive cone. Fields satisfying these conditions are called strongly primitive.

Now let $F$ be a primitive partly ordered field with positive cone $P$, and consider the ring $B / J$, with elements $\bar{x}, \bar{y}$, etc. If $\bar{x}$ contains an element $a \in F$ such that $a+1 / n>0$ holds for every $n=1,2, \cdots$, then we write " $\bar{x}>\overline{0}$ " and use $\bar{P}$ to denote the set of all such $\bar{x}$. If $\bar{x}$ 
belongs to $\bar{P}$ and $b$ is any element in $\bar{x}$ then $b+1 / n>0$ holds for $n=1,2, \cdots$. Note that $\overline{0} \in \bar{P}$; if $\bar{x},-\bar{x}$ both belong to $\bar{P}$ then $\bar{x}=\overline{0}$, and if $\bar{x}+(\overline{1} / \bar{n})>\overline{0}$ for $n=1,2, \cdots$, then $\bar{x} \geqq \overline{0}$. Also, for every $\bar{x}$ there is an integer $n$ such that $\bar{n}-\bar{x}>\overline{0}$.

The norm of $\bar{x}$, written $\mu(\bar{x})$ is defined as follows:

$$
\mu(\bar{x})=\inf \{r ;-\bar{r}<\bar{x}<\bar{r}\},
$$

where $r$ is a positive rational. $\mu(\bar{x})$ satisfies:

(1) $\mu(\bar{x}) \geqq 0 ; \mu(\bar{x})=0$ if and only if $\bar{x}=\overline{0}$.

(2) $\mu(\bar{x})+\mu(\bar{y}) \geqq \mu(\bar{x}+\bar{y})$.

(3) $\mu(\bar{x}) \cdot \mu(\bar{y}) \geqq \mu(\bar{x} \bar{y})$.

(4) $\mu(\bar{r} \bar{x})=|r| \mu(\bar{x})$, for rational $r$.

(5) $\mu(\overline{1})=1$.

The completion of $B / J$ relative to the norm $\mu(\bar{x})$ will be designated by $B^{*}$, with elements $x^{\prime}, y^{\prime}, \cdots$. Let $P^{*}$ be the set of all elements of $B^{*}$ which are limits of elements of $\bar{P}$. Since $\mu(\bar{r})=|r|$ for rational $r$, $P^{*}$ contains the positive real numbers. In fact $B^{*}$ is a complete archimedean (partly) ordered algebra as defined by Kadison [4] and $P^{*}$ is an extension of $\bar{P}$ such that $P^{*} \cap B / J=\bar{P}$. The theorem of Kadison [4] (see also Stone [6]) now gives the structure theorem.

THEOREM 2.2. $B^{*}$ is isomorphic and isometric with the algebra $C(\mathfrak{T C})$ of all real-valued continuous functions on a compact Hausdorff space $\mathfrak{T}$, and $P^{*}$ corresponds biuniquely with the set of non-negative functions in $C(\mathfrak{T T})$; the metric on $C(\mathfrak{T T})$ is given by the sup norm

$$
\|x\|=\sup \{|x(M)| ; M \in \mathfrak{N}\} \text {. }
$$

The elements of $\mathscr{T}$ are the maximal ideals in $B^{*}$, and $x^{\prime} \in B^{*}$ corresponds to the real function $\hat{x}^{\prime}(M)$ which assumes for each $M$ the (unique) real number belonging to the same residue class as $x^{\prime}$.

Corollary 1. If $F$ is $(A C)$ then $x \geqq 0$ holds for $x$ in $B$ if and only if $\hat{x}(M)$ is a non-negative function.

Proof. Since $J$ is the null ideal, $B / J=B$ and $x+1 / n>0$ for $n=1,2, \cdots$ implies $x \geqq 0$. Hence $\bar{P}$ consists of $P \cap B$ and zero.

CoRollary 2. If $\sum x_{i}^{2}$ belongs to $J$, for $x_{i}$ in $B$, then each $x_{i}$ belongs to $J$.

3. Archimedean conditions. An ordered field is called archimedean if every element $x$ is less than some integer $n=n(x)$. If the same condition holds in a partly ordered field $F$ we shall say that $F$ is archimedean in the strong sense, or (AS).

TheOREM 3.1. Let $F$ be $(A S)$. Then $F$ is $(A C)$ and primitive and every 
primitive partly ordered algebraic extension ${ }^{2} F^{\prime}$ of $F$ is also $(A S)$.

Proof. Since every element of $F$ can be expressed as the difference of two positive elements, every additive subgroup of $F$ that contains $P$ also contains $F$. Hence $F$ is primitive. If $x+1 / n>0$ holds for $n=1,2, \cdots$ and if $x \neq 0$, then there is a positive integer $k$ such that $k+1 / x$ and $x+1 / k$ are both positive. Hence $x=k(x+1 / k) /(k+1 / x)$ is positive, so $F$ is (AC).

Let $B^{\prime}$ be the ring of bounded elements of $F^{\prime}$. Then we have $B=F \subset B^{\prime} \subset F^{\prime}$. But since $F^{\prime}$ is algebraic (finite or infinite) over $F, B^{\prime}$ is a field which, by primitivity of $F^{\prime}$, is equal to $F^{\prime}$. Hence $F^{\prime}$ is (AS).

The first two examples of $\$ 1$ are primitive and (AC), but not (AS).

If $F$ is a primitive (AC) field, the ideal $J$ is null so that $B / J=B$. By Theorem 2.1 and Corollary 1 to Theorem 2.2, $x^{2} \geqq 0$ holds for every $x$ in $F$. The following is quite analogous to Baer [3, Satz 4].

LEMMA. If $x^{2} \geqq 0$ holds for every $x$ in an arbitrary partly ordered field $F$, then the partial order is a product of simple orderings.

Proof. Let $P^{\prime}$ be any positive cone in $F$ containing all squares and let $x \neq 0$. A polynomial $f(x)$ in $x$ with coefficients in $P^{\prime}$ can be written: $\sum p_{i} x^{i}=p+q x$ with $p$ and $q$ in $P^{\prime}$. Hence, if $f(x)=0,-x=p / q$ belongs to $P^{\prime}$. Thus if $x$ is any nonzero element such that neither $x$ nor $-x$ belongs to $P^{\prime}$, the set $P^{\prime \prime}$ of all elements of $F$ which can be expressed as rational functions in $x$ with coefficients in $P^{\prime}$ forms a positive cone that properly contains $P^{\prime}$. It follows at once that any positive cone containing all squares is the intersection of all maximal positive cones containing it (such cones exist, by Zorn's lemma) and that each maximal cone gives a simple ordering of $F$.

The following theorem is a simple consequence of the lemma:

TheOREM 3.2. A necessary and sufficient condition that a partial ordering $S$ of $F$ be primitive and $(A C)$ is that $S=\prod S_{\alpha}$ where each $S_{\alpha}$ is a simple ordering, such that if $x$ is positive or infinitesimal according to every $S_{\alpha}$, then $x$ is positive (by $S$ ).

Throughout the rest of the section, $F$ will denote a primitive (AC) field with positive cone $P$, and ring $B$ of all bounded elements.

Let $S=\left\{S_{\alpha}\right\}_{\alpha \in \mathfrak{Q}}$ be the family of all simple orderings whose positive cones $P_{\alpha}$ contain $P$. Some or all of the $S_{\alpha}$ may be non-archimedean. The ring $B$ and ideal $J$ corresponding to $S_{\alpha}$ are denoted by $B_{\alpha}$ and $J_{\alpha}$, respectively, and $\mathscr{T}=\{M\}$ is the family of all maximal ideals in the ring $B^{*}$, the completion of $B . S_{\alpha}$ and $M$ are said to be

\footnotetext{
${ }^{2}$ This is understood to mean that $F^{\prime}$ preserves the partial order of $F$.
} 
associated if $P_{\alpha}$ contains every element $x$ of $B$ such that $\hat{x}(M)>0$. Two ideals associated with the same ordering $S_{\alpha}$ are identical. If $S_{\alpha}$ has an associated ideal it will be denoted by $M_{\alpha}$. Each of the following conditions is necessary and sufficient that $S_{\alpha}$ and $M_{\alpha}$ be associated: (i) For every $x$ in $B \cap P_{\alpha}, \hat{x}\left(M_{\alpha}\right) \geqq 0$; (ii) If $\hat{x}\left(M_{\alpha}\right) \geqq 0$ for $x$ in $B$, then $x \in J_{\alpha} \cup P_{\alpha}$. Also, if $S_{\alpha}$ and $M_{\alpha}$ are associated then $M_{\alpha} \cap B$ $=J_{\alpha} \cap B$.

Two orderings $S_{\alpha}$ and $S_{\beta}$ in $\mathcal{S}$ are called equivalent if $J_{\alpha}=J_{\beta}$, $B_{\alpha}=B_{\beta}$ and both $S_{\alpha}$ and $S_{\beta}$ induce the same ordering in $B_{\alpha} / J_{\alpha}$ $=B_{\beta} / J_{\beta}$. For example, if $x$ is positive and infinitesimal by $S_{1}$ in $K(x)$, negative and infinitesimal by $S_{2}$, then $S_{1}$ and $S_{2}$ are equivalent. However, we only use the notion of equivalence for orderings of $F$ that belong to the family $s$. Two orderings associated with the same ideal are equivalent; and if $S_{\alpha}$ is associated with $M_{\alpha}$ and $S_{\beta}$ is equivalent to $S_{\alpha}, S_{\beta}$ is also associated with $M_{\alpha}$. We say that $M$ is associated with an equivalence class of orderings if it is associated with any (hence with each) ordering in the class. The equivalence class containing $S_{\alpha}$ is denoted by $\bar{S}_{\alpha}$.

Suppose an element $x$ of $F$ were positive by each $S_{\alpha} \in J$ where $J$ is a subfamily of $\mathcal{S}$ containing at least one element of each equivalence class. Then $x$ is positive. For let $S_{\beta}$ be an arbitrary element of $\mathcal{S}$. There is an ordering $S_{\alpha}$ in $\mathfrak{J}$, equivalent to $S_{\beta}$, and $x$ is positive according to $S_{\alpha}$ by hypothesis. Hence $x$ is either positive or infinitesimal according to $S_{\beta}$. Thus $x$ is either positive or infinitesimal by every ordering in $\delta$, so that, by Theorem $3.2, x$ is positive by every ordering in $s$; i.e., $x$ is positive. Thus, the family $J$ gives the same partial order as $\delta$.

LEMMA 1. Every equivalence class of orderings is associated with a maximal ideal $M$.

Proof. For each $\alpha, B_{\alpha} / J_{\alpha}$ is order-isomorphic with a subfield of the field $R$ of all real numbers. For $x$ in $B$, let $\bar{x}(\alpha)$ be the real image of the residue class in $B_{\alpha}$ containing $x$. For $S_{\alpha}$ equivalent to $S_{\beta}$, $\bar{x}(\alpha)=\bar{x}(\beta)$ holds for every $x$. For rational $r$ and arbitrary $x$ in $B$, $r \geqq x$ holds in $F$ if and only if $r \geqq \bar{x}(\alpha)$ holds for every $\alpha$. Hence the norm $\mu(x)$ is equal to sup $\{|\bar{x}(\alpha)| ; \alpha \in \mathfrak{A}\}$. Let $x$ be any element of $B^{*}$. If $\left\{x_{n}\right\}$ is any sequence of elements of $B$ converging to $x$, then for each $\alpha,\left\{\bar{x}_{n}(\alpha)\right\}$ is a convergent sequence of real numbers whose limit $L(x, \alpha)$ is independent of the choice of sequences converging to $x$; moreover, if $x$ belongs also to $B$, then $L(x, \alpha)=\bar{x}(\alpha)$. For arbitrary $x$ in $B^{*}$, let $\bar{x}(\alpha)$ be defined by: $\bar{x}(\alpha)=L(x, \alpha)$. Now $r \geqq x$, for real $r$, holds in $B^{*}$ if and only if $r \geqq \bar{x}(\alpha)$ holds for every $\alpha$, so that $\mu(x)$ $=\sup \{|\bar{x}(\alpha)| ; \alpha \in \mathfrak{A}\}$. 
For fixed $\alpha$, the mapping $H: x \rightarrow \bar{x}(\alpha)$ gives a homomorphism of $B^{*}$ onto the field $R$, whose kernel is a maximal ideal $M_{\alpha}$ in $B^{*}$, with $\hat{x}\left(M_{\alpha}\right)=\bar{x}(\alpha)$. For $x$ in $B \cap P_{\alpha}$, we have $\hat{x}\left(M_{\alpha}\right)=\bar{x}(\alpha) \geqq 0$ so that $M_{\alpha}$ is associated with $S_{\alpha}$, and hence with the equivalence class $\bar{S}_{\alpha}$ containing $S_{\alpha}$.

LEMma 2. Let $\mathcal{K}$ be a set of maximal ideals of $B^{*}$. The following condition is necessary and sufficient that $M^{\prime}$ be a limit point of $\mathfrak{K}$ (in the topology of $\mathfrak{T}$ mentioned in Theorem 2.2): If $\hat{x}(M) \geqq 0$ holds for every $x$ in $B$ and every $M$ in $\mathcal{K}$ (except $\left.M^{\prime}\right)$, then $\hat{x}\left(M^{\prime}\right) \geqq 0$.

Proof. Necessity follows from the fact that the functions $\hat{x}(M)$ are continuous on $\mathfrak{T}$. Sufficiency is a result of Urysohn's lemma, since $B$ is dense in $B^{*}$.

Let $\mathfrak{T}_{s}$ denote the family of all maximal ideals that are associated with orderings in $S$.

Lemma 3. Every $M^{\prime}$ in $\mathfrak{T l}$ is either a limit point of $\mathfrak{T H}_{s}$, or a member of $\mathfrak{T H}_{\mathrm{s}}$ and associated with an archimedean ordering.

Proof. Let $x$ be a member of $B$ such that $\hat{x}(M) \geqq 0$ for every $M$ in $\mathfrak{M}_{s}$ except $M^{\prime}$. If $M^{\prime}$ does not belong to $\mathfrak{M}_{s}$ then $x$ is positive or infinitesimal by every ordering in $\delta$, and therefore positive in $F$. It follows that $\hat{x}(M) \geqq 0$ holds for every $M$ in $\mathfrak{T}$. This implies that $M^{\prime}$ is a limit point of $\mathfrak{T}_{8}$. If $M^{\prime}$ is associated with $S_{\alpha}$, where $S_{\alpha}$ is nonarchimedean, then $x$ is positive or infinitesimal by every $S_{\beta}$ in $\delta$, except possibly $S_{\alpha}$. But then $x y^{2}$ is positive or infinitesimal by every $S_{\beta}$, where $y$ is a nonzero element of $B \cap J_{\alpha}$, so that $x y^{2} \geqq 0$. Hence $x$ is positive in $F$ so that $\hat{x}\left(M^{\prime}\right) \geqq 0$ holds. Again $M^{\prime}$ is a limit point of $\mathfrak{T}_{s}$.

TheOREM 3.3. I. B satisfies the condition: $(\alpha)$ If $\hat{x}(M)$ vanishes on a nonempty open set then $\hat{x}(M)$ vanishes identically. II. Conversely, let $C(T)$ be the ring of all real-valued continuous functions on an arbitrary compact Hausdorff space $T$ and suppose $A$ is a dense subring of $C(T)$ satisfying $(\alpha)$ and $(\beta)$ : If $\{a(t) / b(t) ; t \in T, b(t) \neq 0\}$ is a bounded set of reals, then the equation $b x=a$ has a solution $x \in A$. ( $a$ and $b$ are assumed to belong to $A$.) Then the field $\bar{A}$ of quotients of $A$ has a primitive $(A C)$ partial order by which $A$ is the ring of bounded elements, and $T$ is homeomorphic with the space of maximal ideals in $A^{*}$.

Proof. I. Suppose $\hat{x}(M)$ vanishes for every $M$ in the open set $\mathcal{G}$. If $\mathcal{G}$ contains an $M_{\alpha}$ associated with an archimedean $S_{\alpha}$ the conclusion is obvious. If $\mathcal{G}$ does not contain such an $M_{\alpha}$ then $\mathcal{G}$ contains a limit point of $\mathfrak{M}_{s}$ and hence there is an $M_{\beta} \in \mathcal{G}$ associated with a nonarchimedean $S_{\beta}$. Since $M_{\beta}$ is not a limit point of the complement of 
$\mathcal{G}$, there is an element $y$ in $B$ such that $\hat{y}(M) \geqq 0$ outside $\mathcal{Q}$ and $\hat{y}\left(M_{B}\right)<0$. It follows that $\hat{y} \hat{x}^{2}$ is non-negative on all of $\mathfrak{M}$ so that $y x^{2} \geqq 0$. If $x$ were not zero, $y x^{2}$ would be negative by $S_{B}$, since $y$ is. But this contradicts $y x^{2} \geqq 0$. Hence $x$ is zero.

II. Let $P^{*}$ be the set of all non-negative functions in $A$ (except the zero function) and let $P$ be the set of quotients $a / b$ such that $a b$ belongs to $P^{*}$. The conditions that $A$ be dense in $C(T)$ and $(\beta)$ are not necessary to deduce that $P$ defines an (AC) partial order of $\bar{A}$. The first simply guarantees that the space $T$ is homeomorphic with the space of maximal ideals in $A^{*}$, and the second assures that $A$ contains all bounded elements of $\bar{A}$. The condition $(\alpha)$, besides implying that $A$ is an integral domain, implies that $A$ satisfies the condition that if $a(t)$ and $a(t) b(t)$ belong to $P^{*}$ then $b(t) \in P^{*}$. From this last fact, together with the obvious properties that $P^{*}$ is closed under addition and multiplication and contains all squares in $A$, and that $A$ satisfies: $n a(t)+b(t) \in P^{*}$ for $n=1,2, \cdots$ implies $a(t) \in P^{*}$, the conclusion of the converse is readily deduced. The details are omitted.

Let $\mathcal{S}^{*}$ designate the family of all equivalence classes in $\delta$, with the class containing $S_{\alpha}$ denoted by $\bar{S}_{\alpha}$. The $L^{*}$ topology in $\mathcal{S}^{*}$ is defined as follows [ $\bar{x}$ is defined in Lemma 1]:

$\left(L^{*}\right) \bar{S}_{\alpha}$ is a limit point of a set $\mathfrak{K} C S^{*}$ if and only if for every $x$ in $B$, $\bar{x}(\beta) \geqq 0$ for every $\bar{S}_{\beta}$ (except $\bar{S}_{\alpha}$ ) in $\pi$ implies $\bar{x}(\alpha) \geqq 0$.

TheOREM 3.4. With the topology $\left(L^{*}\right), s^{*}$ is homeomorphic with Tr.

Proof. It is already clear that $\delta^{*}$ is homeomorphic with $\mathfrak{T l}_{s}$, so that we have left to show only that $\mathfrak{T C}_{s}=\mathfrak{N}$, i.e., that every maximal ideal is associated with an ordering in $\delta$.

Let $M_{1}$ be an arbitrary element of Tr. Let $P_{1}$ be a subset of $B$ defined by: a nonzero element $x$ of $B$ belongs to $P_{1}$ if there is an open set $\mathcal{G}_{x}$ (containing $M_{1}$ ) in $\mathfrak{T}$, such that $\hat{x}(M)$ is non-negative on $\mathcal{S}_{x}$. We have:

(i) $P_{1}$ is closed under addition and multiplication.

(ii) For every nonzero $x, x^{2} \in P_{1}$.

(iii) If $x$ and $x y$ belong to $P_{1}$, then $y$ belongs to $P_{1}$. For both $\hat{x}(M)$ and $\hat{x} \hat{y}(M)$ are non-negative on $\mathcal{G}=\mathcal{G}_{x} \cap \mathcal{G}_{x y}$. If $\hat{y}\left(M^{\prime}\right)<0$ holds for any $M^{\prime}$ in $\mathcal{G}$ then $\hat{y}(M)$ is negative throughout an open set $\mathcal{G}^{\prime} \subset \mathcal{G}$. But then $\hat{x}(M)$ must vanish on $\mathcal{G}^{\prime}$, whence, by Theorem 3.2, $x=0$. This contradicts $x \in P_{1}$ so that $\hat{y}(M)$ is non-negative on $g ;$ hence $y \in P_{1}$.

(iv) If $\hat{x}\left(M_{1}\right)>0$, then $x \in P_{1}$.

(v) $P_{1}$ contains $P \cap B$.

Properties (i), (ii) and (iii) permit us to define a positive cone $\bar{P}_{1}$ 
in $F$ as the set of all quotients $x / y(x$ and $y$ in $B$ ) for which $x y$ belongs to $P_{1}$. Then by (ii) and the lemma preceding Theorem $3.2, \bar{P}_{1}$ may be extended to at least one simple cone, which contains $P$, by $(v)$. If $\widetilde{P}_{1}$ is any simple extension of $\bar{P}_{1}$, then the ordering corresponding to $\tilde{P}_{1}$ belongs to $S$, and from (iv) is associated with $M_{1}$. Thus $M_{1}$ is associated with the equivalence class of all simple extensions of $\bar{P}_{1}$. This completes the proof.

Theorem 3.5. $B=\bigcap_{\alpha \in \mathfrak{q}} B_{\alpha}$.

Proof. It is obvious that $B$ is contained in $\cap B_{\alpha}$. Let $x$ be any element not belonging to $B$. There exists a sequence $\left\{\alpha_{k}\right\}$ such that $x^{2}>k$ holds by $S_{\alpha_{k}}, k=1,2, \cdots$. The element $1 /\left(1+x^{2}\right)$, which belongs to $B$ in any case, consequently has no inverse in $B$ and is therefore a member of a maximal ideal $M_{\alpha}$ associated with an ordering $S_{\alpha}$. It follows that, since $1 /\left(1+x^{2}\right)$ belongs to $J_{\alpha}, 1+x^{2}$ is not in $B_{\alpha}$. Hence $x$ is not in $B_{\alpha}$, and consequently is not in $\cap B_{\alpha}$.

According to Krull [5], the intersection of integrally closed domains is integrally closed. Clearly every $B_{\alpha}$ is integrally closed.

CoROllary. $B$ is integrally closed.

4. Extensions. Let $F$ and $F^{\prime}$, with $F \subset F^{\prime}$, be partly ordered with positive cones $P$ and $P^{\prime}$. If $P^{\prime} \cap F=P$, we shall say that the partial order of $F$ has been extended to $F^{\prime}$, or that the partial order of $F^{\prime}$ preserves that of $F$. First we consider simple algebraic extensions. Let $F$ be primitive, $\theta$ a root of the monic irreducible polynomial $f(x)$ $=\sum_{i=0}^{n} f_{i} x^{i},(n>1)$ with coefficients in $B$, and suppose that $J$ is not the null ideal in $F$. In $F^{\prime}=F(\theta)$ define $P^{\prime}$ as the set of all elements $\sum_{i=0}^{n-1} a_{i} \theta^{i}$ for which $a_{0} \in P$, and $a_{i} / a_{0} \in J$ for $i=1,2, \cdots, n-1$. We wish to show that $P^{\prime}$ is a positive cone.

Lemma 1. If $a_{i} / a_{0} \in J$ for $i=1,2,3$ then $\left(a_{1}+a_{2}\right) /\left(a_{3}+a_{0}\right) \in J$.

Proof. Note that $1+a_{3} / a_{0}$ is a unit in $B$. Hence $\left(a_{1}+a_{2}\right) /\left(a_{3}+a_{0}\right)$ $=\left(a_{1} / a_{0}+a_{2} / a_{0}\right) /\left(1+a_{3} / a_{0}\right)$ belongs to $J$.

Since $f(x)$ is monic, all powers of $\theta$ have representations with coefficients in $B$ of the form: $\theta^{k}=\sum_{i=0}^{n-1} b_{l}^{(k)} \theta^{i}$.

Lemma 2. $P^{\prime}$ contains every element of the form $y=\sum_{i=0}^{m} a_{i} \theta^{i}$, where $a_{0} \in P, a_{i} / a_{0} \in J$ for $i=1,2, \cdots, m$.

Proof. $y=\sum_{i=0}^{n-1}\left(a_{i}+\sum_{k=n}^{m} a_{k} b_{i}^{(k)}\right) \theta^{i}$. It follows from Lemma 1 that $y$ belongs to $P^{\prime}$.

COROLLARY. $P^{\prime}$ is closed under addition and multiplication. 
Let $\theta_{1}, \cdots, \theta_{n}=\theta$ be the conjugates of $\theta, \sigma_{k}$ and $\sigma_{k}^{\prime}$ be the $k$ th elementary symmetric functions of $\theta_{1}, \cdots, \theta_{n}$, and $\theta_{1}, \cdots, \theta_{n-1}$, respectively. From the formulas $\sigma_{1}^{\prime}=\sigma_{1}-\theta, \cdots, \sigma_{k}^{\prime}=\sigma_{k}-\theta \sigma_{k-1}^{\prime}$, $k=2, \cdots, n-1$, we see that any symmetric polynomial (with integral coefficients) in $\theta_{1}, \cdots, \theta_{n-1}$ or in $\theta_{1}, \cdots, \theta_{n}$ belongs to $B[\theta]$, the ring of all elements of the form $\sum_{i=0}^{n-1} c_{i} \theta^{i}$, with all $c_{i}$ in $B$. For symmetric polynomials in $\theta_{1}, \cdots, \theta_{n-1}$ we write

$$
\sum \theta_{1}^{i_{1}} \cdots \theta_{k}^{i_{k}}=\sum_{m=0}^{n-1} c_{i_{1} \cdots i_{k}}^{(m)} \theta^{m}
$$

with the $c$ 's belonging to $B$.

Let $y=h(\theta)=\sum_{i=0}^{n-1} h_{i} \theta^{i}$ be an arbitrary element of $P^{\prime}$. The reciprocal of $y$ is $1 / y=h\left(\theta_{1}\right) \cdots h\left(\theta_{n-1}\right) /\left(h\left(\theta_{1}\right) \cdots h\left(\theta_{n}\right)\right)$, where the numerator has the form

$$
h_{0}^{n-1}+\sum_{k=1}^{n-1} h_{0}^{n-1-k}\left\{\sum_{i_{1} \leqq}^{n-1} h_{i_{1}} \cdots h_{i_{k}} \sum \theta_{1}^{i_{1}} \cdots \theta_{k}^{i_{k}}\right\} .
$$

Substituting for the symmetric polynomials from (1), the coefficient of $\theta^{m}$, for $m \geqq 1$, is seen to be a sum of terms of the form

$$
h_{0}^{n-1-k} h_{i_{1}} \cdots h_{i_{k}} c_{i_{1} \cdots i_{k}}^{(m)}
$$

while the constant term is $h_{0}^{n-1}$ plus a sum of terms of the same form. By direct calculation we see that the quotient of each such term by $h_{0}^{n-1}$ belongs to $J$. Hence by Lemmas 1 and 2 , the numerator $h\left(\theta_{1}\right) \cdots h\left(\theta_{n-1}\right)$ belongs to $P^{\prime}$. In the same way it is shown that the denominator belongs to $P$, so that $1 / y$ belongs to $P^{\prime}$. This proves the following:

Lemma 3. $P^{\prime}$ is a positive cone in $F^{\prime}$.

The following theorem is now easily proved:

TheOREM 4.1. The partial order of $F$ may be extended to $F^{\prime}=F(\theta)$ by the positive cone $P^{\prime}$ and this extension preserves primitivity and strong primitivity. If $J$ is a prime ideal, so is $J^{\prime}$. ( $J$ is assumed to be nonnull).

Now let $F$ be an arbitrary primitive partly ordered field and let $F(x)$ be a transcendental extension. Let $f(x)$ be any irreducible polynomial over $F$ and $\theta$ a root. If the partial order of $F$ can be extended to $F(\theta)$ by the positive cone $P_{\boldsymbol{\theta}}$, then it can be extended to $F(x)$ as follows: For arbitrary $g(x) \in F(x)$ write $g(x)=[f(x)]^{\circ} a(x) / b(x)$, where 
$a(x)$ and $b(x)$ are prime to $f(x)$; define $P^{\prime}$ by: $g(x) \in P^{\prime}$ if and only if $a(\theta) / b(\theta) \in P_{\theta}$. $P^{\prime}$ defines a strongly primitive partial order in $F^{\prime}=F(x)$ in every case. For the arbitrary element

$$
g(x)=[f(x)]^{e} a(x) / b(x)
$$

is exceeded by the positive element $[f(x)]^{e-1}$. In particular, $f(x)$ can be chosen to be a linear polynomial $x-\theta$, so that $F(\theta)=F$; then $P^{\prime}$, as defined above, gives a strongly primitive extension to $F(x)$.

TheOREM 4.2. The partial order of $F$ can always be extended to $F(x)$ so that $F(x)$ is strongly primitive.

For an arbitrary extension $G$ of $F$, Zorn's lemma may be applied easily to prove the following:

Theorem 4.3. A partial order of $F$ by which $J$ is not the null ideal can be extended to any comprehending field $G$ over $F$ so as to preserve primitivity, strong primitivity, and the property that $J$ is a prime ideal.

Given a field $F$ with a transcendental (over the rational field $K$ ) element $x, K(x)$ can be ordered so that $J \neq(0)$; by the theorem, any such partial ordering can be extended to $F$.

COROLlARY. If $F$ has any transcendental (over $K$ ) elements then $F$ has a strongly primitive partial order by which $J$ is a prime ideal in $B$.

Finally we shall consider pure transcendental extensions of (AC) fields. Let $F$ be primitive and (AC). The fact that $P$ contains all the squares in $F$ implies that $F$ is strongly primitive. For if $b$ is any element of $F, b^{2}+1$ is a positive element greater than $\mathrm{b}$, since $b^{2}+1-b$ is a sum of squares. It follows that $F$ satisfies the condition $\left(\mathrm{AC}^{\prime}\right)$ : $n x+b>0$ for $n=1,2, \cdots$, implies $x \geqq 0$; in fact, the conditions (AC) and $\left(\mathrm{AC}^{\prime}\right)$ are equivalent for primitive fields.

Let $F^{\prime}=F(x)$ be a simple transcendental extension of $F$ and for each $a$ in $F$, let $P_{a}$ be the set of all functions $(x-a)^{e} f(x) / g(x)$ (with $f(x)$ and $g(x)$ prime to $x-a)$ such that $f(a) / g(a)>0$. It was noted in the proof of Theorem 4.2 that $P_{a}$ is a positive cone in $F^{\prime}$ with $P_{a} \cap F$ $=P$, but $P_{a}$ cannot give an (AC) partial order in $F^{\prime}$. Let $P^{\prime}=\bigcap_{a \in F} P_{a}$.

Theorem 4.4. $P^{\prime}$ defines a primitive $(A C)$ partial order of $F^{\prime}=F(x)$ which preserves the partial order of $F$.

Proof. It is convenient to consider the polynomial ring $F[x]$ and the set $P^{*}=F[x] \cap P^{\prime}$. It is clear that $P^{*}$ is closed under addition, multiplication and cancellation (i.e., if $f$ and $f g$ belong to $P^{*}$ then $\left.g \in P^{*}\right)$, and that $P^{*}$ contains all squares in $F[x]$. In addition, $P^{*}$ 
satisfies $\left(\mathrm{AC}^{\prime}\right)$ : If $n f(x)+g(x) \in P^{*}$ for $n=1,2, \cdots$, where $f(x) \neq 0$ and $f(x)$ and $g(x)$ belong to $F[x]$, then $f(x) \in P^{*}$, as we now show.

Suppose $n f(x)+g(x) \in P^{*}$ for $n=1,2, \cdots$, and assume that $f(x)$ is neither zero nor in $P^{*}$. For every element $b$ of $F$, we have $f(b) \geqq 0$. For if $f(b) \neq 0$, then, since $n f(x)+g(x) \in P_{b}$, we have $n f(b)+g(b) \in P$ for $n=n_{0}, n_{0}+1, \cdots$. Hence $f(b)$ is positive. Since $f(x)$ is not in $P^{*}$ there is an element $a$ in $F$, with $f(x)=(x-a)^{\circ} g(x)$, and $g(a)$ neither positive nor zero. There consequently exists an ordering $S$ of $F$ whose positive cone contains $P$, according to which $g(a)$ is less than a negative rational.

Consider the Taylor's (finite) series

$$
g(a+h)=g(a)+h g^{(1)}(a)+h^{2} g^{(2)}(a) / 2+\cdots .
$$

If $S$ is archimedean the coefficients $g^{(i)}(a)$ are bounded so that $h>0$ can be chosen small enough that $g(a+h)$ is negative by $S$. If $S$ is not archimedean, then for every infinitesimal (by $S$ ) element $y, g(a+h)$ is negative by $S$, where $h=y^{2} /\left(1+\sum\left(g^{(i)}(a)\right)^{2}\right)$. In either case there is an infinite set of positive values of $h$ such that $g(a+h)$ is negative according to $S$ and hence $f(a+h)=h^{e} g(a+h)$ is negative by $S$. For these values of $h, f(a+h)$ is accordingly not positive in $F$. But for every $h$ for which $f(a+h) \neq 0$, we just saw that $f(a+h)$ is positive. Thus $f(x)$, having infinitely many roots, is zero, in contradiction to our assumption. This establishes $\left(\mathrm{AC}^{\prime}\right)$.

Now $P^{\prime}$ is simply the set of all $f(x) / g(x)$ such that $f(x) g(x)$ belongs to $P^{*}$. It is now easy to verify that $P^{\prime}$ is (AC), primitive, and that $P^{\prime} \cap F=P$.

Finally, let $G$ be an arbitrary pure transcendental extension of $F$, by independent indeterminates $x_{\alpha}, \alpha \in \mathfrak{A}$. The positive cone $P^{\prime}$ of the preceding theorem consists of all functions $f(x)$ such that $f(a) \geqq 0$ for every $a$ in $F$ for which $f(a)$ is defined. In order to extend the partial order of $F$ to $G$ we take $\bar{P}$ to be the set of all functions $f\left(x_{\alpha_{1}}, \cdots, x_{\alpha_{n}}\right)$ ( $n$ is arbitrary) such that $f\left(a_{1}, \cdots, a_{n}\right) \geqq 0$ for all $a_{1}, \cdots, a_{n}$ in $F$ for which the function is defined. That this defines a primitive (AC) partial order in $G$ now follows from Theorem 4.4 by finite induction. Thus we have:

Theorem 4.5. The primitive $(A C)$ partial order of $F$ may be extended to an arbitrary pure transcendental extension $G$ of $F$, so that $G$ is also primitive and $(A C)$.

\section{REFERENCES}

1. E. Artin, Über die Zerlegung definiter Funktionen in Quadrate, Abh. Math. Sem. Hamburgischen Univ. vol. 5 (1926) pp. 100-115. 
2. E. Artin and O. Schreier, Algebraische Konstruction reeler Körper, ibid. pp. 83-100.

3. R. Baer, Über nichtarchimedisch geordnete Körper, Sitzungsber. Heidelb. Ak., 8 Abhandlung, 1927.

4. R. V. Kadison, A representation theory for commutative topological algebra, Memoirs of the American Mathematical Society, no. 7, 1951, pp. 1-9.

5. Wolfgang Krull, Allgemeine Bewertungstheorie, Crelle's Journal vol. 167 (1931) pp. $168-169$.

6. M. H. Stone, $A$ general theory of spectra, I, Proc. Nat. Acad. Sci. U.S.A. vol. 26 (1940) pp. 280-283.

UNIVERSITY OF OKLAHOMA AND

Ohio State University 